\title{
Implementing and revitalizing the Baby-Friendly Hospital Initiative
}

\author{
Randa Saadeh and Carmen Casanovas
}

\begin{abstract}
The Baby-Friendly Hospital Initiative (BFHI) was launched in the 1990s by the World Health Organization (WHO) and UNICEF as a global effort with hospitals, health services, and parents to ensure babies are breastfed for the best start in life. It is one of the Operational Targets of the Global Strategy for Infant and Young Child Feeding endorsed in 2002 by the Fifty-Fifth World Health Assembly and the UNICEF Executive Board. After about 18 years, great progress has been made, and most countries have breastfeeding authorities or BFHI coordinating groups. The BFHI has led to increased rates of exclusive breastfeeding, which are reflected in improved health and survival. Based on this progress, the Initiative was streamlined according to the experience of the countries and materials were revised. The new package consolidated all WHO and UNICEF materials into one package, reflected new research and experience, revisited the criteria used for the BFHI in light of HIV/ AIDS, reinforced the International Code of Marketing of Breast-Milk Substitutes, provided modules for motherfriendly care, and gave more guidance for monitoring and reassessment. WHO and partners will continue to give support to BFHI implementation as one essential effort contributing to achievement of the Millennium Development Goals.
\end{abstract}

Key words: Baby-friendly Hospital Initiative, Global Strategy for Infant and Young Child Feeding, Breastfeeding, HIV/AIDS, International Code of Marketing of Breast-milk Substitutes, Mother-friendly care

Randa Saadeh and Carmen Casanovas are affiliated with the World Health Organization, Geneva, Switzerland.

Please direct queries to the corresponding author: Randa Saadeh, Department of Nutrition for Health and Development, WHO, 20, avenue Appia, 1211 Geneva 27, Switzerland. Email: saadehr@who.int

\section{Introduction}

Breastfeeding, especially exclusive breastfeeding, is central to achieving the Millennium Development Goal for child survival. The Baby-Friendly Hospital Initiative (BFHI) is a global effort to implement practices that protect, promote, and support breastfeeding. When the BFHI was launched in the early 1990s in response to the Innocenti Declaration's call for action, there were very few countries that had dedicated, nationallevel, breastfeeding support activities [1]. Today, after nearly 18 years of World Health Organization (WHO) endorsement and UNICEF country-level support for its implementation, most countries have breastfeeding or infant and young child feeding authorities or BFHI coordinating groups and have assessed hospitals and designated at least one facility as "baby-friendly."

The 2002 WHO/UNICEF Global Strategy for Infant and Young Child Feeding [2] includes a call for renewed support - with urgency - for exclusive breastfeeding from birth to 6 months and continued breastfeeding with timely and appropriate complementary feeding for 2 years or longer. At least six of the nine operational targets of the Global Strategy are directly relevant to the BFHI. In addition, step three of the five steps in HIV and Infant Feeding: Framework for Priority Actions [3] is dedicated to improvement of health systems and training of providers, specifically calling for strengthening of the BFHI.

With the renewed call for the BFHI in the Global Strategy for Infant and Young Child Feeding, the challenges posed by the HIV pandemic, and the experiences of countries, WHO and UNICEF led the process through which the global BFHI materials were revised, updated, and expanded for integrated care [4].

\section{Methods}

Since the launching of the BFHI, more than 20,000 hospitals in 156 countries have been designated as baby-friendly. During this time, a number of regional 
meetings offered guidance and provided opportunities for networking and feedback from dedicated country professionals involved in implementing the BFHI. Two of these meetings were conducted in Spain, for the European region, and Botswana, for the Eastern and Southern African region. Both meetings offered recommendations for updating the Global Criteria and related assessment tools, as well as the 18-hour course, in light of experience with the BFHI since the Initiative began, the guidance provided by the new Global Strategy for Infant and Young Child Feeding, and the challenges posed by the HIV pandemic. The importance of addressing mother-friendly care within the Initiative was raised by a number of groups as well.

As a result of the strong interest in the BFHI package and requests for its updating, WHO and UNICEF undertook the revision of the materials in 2004-05, with various people assisting in the process. The process included an extensive user survey among colleagues from many countries. Once the revised course and tools were drafted, they were reviewed by experts worldwide and then field tested in industrialized and developingcountry settings. The full first draft of the materials was posted on the UNICEF and WHO websites as the "Preliminary Version for Country Implementation" in 2006. After more than a year's trial, presentations in a series of regional multicountry workshops, and feedback from dedicated users, UNICEF and WHO met with the coauthors and resolved the final technical issues that had been raised. The final version was completed in late 2007.

The updated materials reflect new research and experience, reinforce the implementation of the International Code of Marketing of Breast-Milk Substitutes, include support to mothers who are not breastfeeding, provide modules on HIV and infant feeding and mother-friendly care, and give more guidance for monitoring and reassessment.

\section{Major recent findings on the impact of the original BFHI package}

New findings underscore the successful implementation of the BFHI worldwide and the impact of the $\mathrm{BFHI}$ on exclusive breastfeeding rates. Studies have also confirmed that exclusive breastfeeding rates increase when a facility becomes baby-friendly, and this increase is reflected in improved health and survival [5]. When included as an essential element in a comprehensive, multisector, multilevel effort to protect, promote, and support optimal infant and young child feeding, the BFHI provides a vital contribution to achieving and sustaining the behaviors and practices necessary to enable every mother and family to give every child the best start in life by creating sustainable change beyond the hospital and throughout the health system.
The impact of the BFHI can be demonstrated at the hospital, community, and country levels.

Evidence from both developed and developing countries indicates that the BFHI has had a direct impact on breastfeeding rates at the hospital level. For example, in Switzerland [6], a 2003 analysis of data from mothers who had given birth in the past 9 months demonstrated that the percentage of infants exclusively breastfed at 0 to 5 months was significantly higher among those who had delivered in baby-friendly hospitals than in the general sample.

The study in Switzerland also noted that the average duration of breastfeeding was longer for those who had given birth in a baby-friendly hospital that showed good compliance with the Ten Steps to Successful Breastfeeding [1] than for those who had given birth in a baby-friendly facility without good compliance, leading investigators to conclude that increases in Switzerland's breastfeeding rates from 1994 to 2003 were attributable in part to the BFHI.

In a randomized, controlled trial in Belarus, Kramer and colleagues noted improved rates of exclusive breastfeeding and any breastfeeding long after the hospital stay, as well as reduced rates of illness, among infants of mothers giving birth at hospitals randomly assigned to follow BFHI policies compared with those delivering at control hospitals [7].

In an urban area of Montes Claros in Brazil, a population area served by three hospitals that were designated as baby-friendly there was a much more rapid increase in initiation and duration of exclusive breastfeeding than in surrounding areas [8].

Although global trends in breastfeeding initiation, duration, and exclusivity have been generally increasing during the years since BFHI implementation, few studies have examined the association between trends in BFHI activities at the national level and improvements in breastfeeding practices. One study conducted in Sweden noted that the percentage of mothers breastfeeding at 6 months increased dramatically from $50 \%$ at the start of the country's BFHI in 1993 to $73 \%$ in 1997 , when $100 \%$ of maternity centers had achieved baby-friendly status, a result suggesting that the BFHI was effective in promoting breastfeeding among Swedish mothers [9]. However, trends in breastfeeding have resulted from multiple interventions, policy shifts, and changing social norms, making it difficult to separate impacts specific to this Initiative.

\section{Lessons learned and identified gaps}

Country reports presented to WHO and UNICEF found that:

"The BFHI is doable. All country reports and studies confirmed that programs to implement the BFHI, following the guidance provided, resulted in an 
increase in the desired practices.

"The BFHI is adaptable. The BFHI was appropriate for countries with vastly differing circumstances and was found to be extremely adaptable, as it has now been implemented in a wide variety of cultural and socioeconomic settings.

" The BFHI may enhance attention to quality of care for others, based on health worker interviews. Many health workers and hospital administrators have noted that the BFHI was a catalyst to considering quality of care in other hospital sectors.

" BFHI sustainability demands ongoing political and financial commitment to quality. Insufficient recognition of the effectiveness and impact of the BFHI by policy makers, health professionals, and donors can result in a diminution of quality and sustainability.

" The BFHI will not be fully sustainable until infant and young child feeding support skills, including the techniques of breastfeeding support, are fully integrated into preservice health worker education. The need for reassessments and refresher training demands constant investment, which could be reduced by strengthening in-service curricula and training for all health workers.

"Step 10-which is the only step that extends beyond hospital settings and concerns community protection, promotion, and support for breastfeeding - is not well implemented and linked to the facility effort, ensuring support to mothers after discharge, and thus resulting in reduced impact. Community support-through skills training and social marketing-is a vital link to an effective and sustainable program, whether initially developed as part of the BFHI or integrated into ongoing community activities.

In assessing the overall feedback from and status of BFHI implementation in countries, it was evident that although no basic changes were necessary in the BFHI, there was an urgent need to address maintenance of quality, sustainability, integration and mainstreaming, expansion, including preservice training, implementation of step 10, and awareness of feeding plans of HIV-positive women.

\section{Revised package}

The revised BFHI package has five sections:

Section 1: Background and Implementation provides guidance on the revised processes, sustainability, integration, and expansion options at the country, health facility, and community levels, recognizing that the Initiative has expanded and must be mainstreamed to some extent for sustainability. It includes subsections on country- and hospitallevel implementation; the Global Criteria for the BFHI; compliance with the International Code of Marketing of Breast-Milk Substitutes; baby-friendly expansion and integration options; and resources, references, and websites.

Section 2: Strengthening and Sustaining the BabyFriendly Hospital Initiative: A Course for Decision-Makers was adapted from the WHO course "Promoting breast-feeding in health facilities: a short course for administrators and policy-makers." It can be used to orient hospital decision makers (directors, administrators, key managers, etc.) and policy makers to the Initiative and the positive impacts it can have, and to gain their commitment to promoting and sustaining the Ten Steps. There are a Course Guide and eight session plans with handouts and PowerPoint slides. Two alternative session plans and materials for use in settings with high HIV prevalence have been included.

Section 3: Breastfeeding Promotion and Support in a Baby-Friendly Hospital, a 20-hour course for maternity staff, can be used by facilities to strengthen the knowledge and skills of their staff for successful implementation of the Ten Steps to Successful Breastfeeding and the International Code of Marketing of Breast-Milk Substitutes. This course replaces the 18-Hour Course. It includes guidelines for course facilitators, outlines of course sessions, and PowerPoint slides for the course. The section also suggests an agenda and content for training nonclinical staff.

Section 4: Hospital Self-Appraisal and Monitoring provides tools that can be used by managers and staff initially to help determine whether their facilities are ready to apply for external assessment and, once their facilities are designated baby-friendly, to monitor continued adherence to the Ten Steps. This section includes a hospital self-appraisal tool and guidelines and tools for monitoring.

Section 5: External Assessment and Reassessment provides guidelines and tools for external assessors to assess initially whether hospitals meet the Global Criteria and thus fully comply with the Ten Steps and then to reassess, on a regular basis, whether they continue to maintain the required standards. This section includes a guide for assessors, a hospital external assessment tool, PowerPoint slides for training assessors, guidelines and tools for external reassessment, and a computer application for analyzing results.

The revised package of BFHI materials is available on the WHO and UNICEF websites. Sections 1 through 4 can be accessed at: www.unicef.org/nutrition/index_24850.html, or at: www.who.int/nutrition/ topics/bfhi/en/index.html.

Section 5, External Assessment and Reassessment, is not available for general distribution. It is available to the regional and national UNICEF officers through the UNICEF Intranet and at WHO regional offices to provide to the national authorities for the BFHI. It is 
also made available to the assessors who conduct BFHI assessments and reassessment.

\section{The major updates and revisions include the following:}

"An updated and enhanced set of guidelines for implementing the BFHI at the country and hospital levels, including options for enhancing sustainability, further integrating the BFHI into the health-care system, and expanding its focus into other health units and into the community.

» Additional content related to:

- IV and infant feeding in the two courses (sections 2 and 3) and an optional HIV and infant-feeding module in the self-appraisal, monitoring, and assessment tools (sections 4 and 5).

- Mother-friendly childbirth practices in the 20-hour course and a new "mother-friendly care" module in the self-appraisal, monitoring, and assessment tools.

- The International Code of Marketing of BreastMilk Substitutes in the two courses and a new "Code" module in the monitoring and assessment tools.

- Support for non-breastfeeding mother-baby pairs in the 20-hour course and added material in the self-appraisal, monitoring, and assessment tools to assess the support for these mothers and their infants.

"An update in the "Medical Reasons for Use of Breast-Milk Substitutes." The list is now available as an attachment to the BFHI material and as an independent document [10].

»Inclusion of updated technical information and data from recent research in the two courses and an updated set of references, including websites, in section 1.

"An enhanced practical focus in the 20-hour course, with the addition of a story of two mothers and role-playing and problem-solving exercises related to their support, and additional clinical practice sessions.

" An upgraded set of assessment tools, with improved key points, added guidance for sampling and tallying results to assist assessors, etc.

"A new self-contained computer-based tool for tallying and presenting assessment results that can be used without any additional software.

" PowerPoint slides and transparency templates for use with the two courses and for training assessors.

" Additional guidance and tools for use in monitoring and reassessment of baby-friendly health-care facilities in sections 4 and 5.

In addition, the updated materials provide guidance on the road to baby-friendly designation at the health facility level. The following points are suggested:
" If your health facility is not yet designated as babyfriendly this is what to do:

- Obtain the BFHI self-appraisal materials from your country's central BFHI coordination group and complete the self-appraisal.

- Make an action plan to address any areas that need attention. Find out what support is available from your country's central BFHI coordination group.

- Develop and undertake a comprehensive training plan to address the needs that will lead to changes in practices supporting the baby-friendly process.

- When the self-appraisal indicates a high standard of practice, contact your country's central BFHI coordination group to arrange for an external assessment team to visit the health facility.

- When the health facility is designated as babyfriendly, carry out ongoing monitoring or auditing to ensure that the practices remain supportive.

" If your health facility was designated baby-friendly more than 3 years ago, there may need to be a reassessment to ensure the practices are still in place. Follow the steps for the original assessment: self-appraisal, action planning as needed, seeking external assessment, and then ongoing monitoring or auditing.

\section{The way forward}

WHO and UNICEF have policies and strategies in place that outline many of the necessary steps in the way forward, and should continue to support those plans. Governments should ensure that all personnel who are involved in health, nutrition, child survival, or maternal health are fully informed and energized to take advantage of an environment that is conducive for revitalizing the BFHI; incorporate the basic competencies for protection, promotion, and support of optimal infant and young child feeding, including the BFHI, into all health-worker curricula, whether facility- or community-based health workers; recognize that the BFHI has a major role to play in child survival in general and more so in the context of HIV/AIDS; and seek and establish regional collaboration for skills development; seek funding and political support as well as commitment on the part of all UN agencies and governments to revitalize the BFHI in order to achieve Millennium Development Goals.

Decision and policy-makers should be sensitized and encouraged to support the BFHI as an essential part of a comprehensive infant and young child feeding approach. The Innocenti +15 provides a list of priority actions that include the BFHI [11]. However, the sensitization, or social marketing, for decision makers is a significant undertaking. Country-to-country support and the training of decision makers using the provided 
tools are two proven options.

Step 10, or community action to protect, promote, and support breastfeeding, must be strengthened. Mainstreaming and integrating breastfeeding into community programs must be carried out in a manner that sustains the good quality of support. This is not simply a matter of community information, but rather necessitates good-quality training, social marketing, community mobilization, and backup from a prepared health system.

The Planning Guide for implementation of the Global Strategy for Infant and Young Child Feeding [12] may provide additional approaches and ideas for exciting and energetic implementation planning. It is important to build upon what exists and to create a situation where there is sustainability of all four pillars: legislation and regulatory support, health-worker education, health-system monitoring and ongoing assessment, and community action may be the key to a comprehensive and sustainable cost-effective initiative.
Throughout, the focus should remain on improved quality and impact and the shared global goal of increasing maternal, infant, and child survival.

\section{Conclusions}

The BFHI has had great impact on breastfeeding practices. Learning from countries' experience in implementing this Initiative and reflecting new infantfeeding research findings and recommendations, the tools and courses used to change hospital practices in line with baby-friendly criteria have been streamlined, updated, and revised and put into one set of materials. WHO and UNICEF strongly recommend using this new set of materials to ensure solid and full implementation of the BFHI global criteria and sustain progress already made. It is one way of improving child health and survival and moving ahead to meet the Millennium Development Goals.

\section{References}

1. Protecting, promoting and supporting breast-feeding: The special role of maternity services. A joint $\mathrm{WHO} /$ UNICEF statement. Geneva: WHO, 1989.

2. World Health Organization/UNICEF. Global strategy for infant and young child feeding. Geneva: WHO, 2003.

3. Joint United Nations Programme on HIV/AIDS/Food and Agriculture Organization/United Nations High Commissioner for Refugees/UNICEF/World Health Organization/World Food Programme/World Bank/ United Nations Population Fund/International Atomic Energy Agency. HIV and infant feeding: Framework for priority actions. Geneva: WHO, 2003.

4. World Health Organization/UNICEF. Baby-Friendly Hospital Initiative: Revised, updated and expanded for integrated care. Geneva: WHO, 2008.

5. Perez-Escamilla R. Evidence based breast-feeding promotion: The Baby-Friendly Hospital Initiative. J Nutr 2007;137:484-7.

6. Merten S, Dratva J, Ackermann-Liebrich U. Do BabyFriendly Hospitals influence breastfeeding duration on a national level? Pediatrics 2005:116:e702-8.

7. Kramer MS, Chalmers B, Hodnett ED, Sevkovsata Z, Dzikovich I, Shapiro S, Collet JP, Vanilovich I, Mezen I, Ducruet T, Shishko G, Zubovich V, Mknuik D, Gluchanina E, Dombrovskiy V, Ustinovitch A, Kot T, Bodganovich N,
Ovchinikova L, Helsin E. Promotion of Breastfeeding Intervention Trial (PROBIT): A randomized trial in the Republic of Belarus. JAMA 2001;285:413-20.

8. Caldeira AP, Gonzales E. Assessment of the impact of implementing the Baby-Friendly Hospital Initiative. J Pediatr (Rio J) 2007;83:127-32.

9. Hofvander Y. Breastfeeding and the Baby Friendly Hospitals Initiative (BFHI): Organization, response and outcome in Sweden and other countries. Acta Paediatr Scand 2005;94:1012-6.

10. World Health Organization/UNICEF. Acceptable medical reasons for use of breast-milk substitutes. Geneva: WHO, 2008.

11. Academy for Education and Development (AED)/ Intenational Baby Food Action Network (IBFAN)/ International Lactation Consultant Association (ILCA)/ La Leche League International (LLLI)/Regione Toscana/ UNICEF/Wellstart International/World Alliance for Breastsfeeding Action (WABA) World Health Organization. Innocenti Declaration 2005 on infant and young child feeding. 22 November 2005. Florence, Italy Innocenti, UNICEF Innocenti Research Center, 2006.

12. World Health Organization/UNICEF. Planning guide for national implementation of the Global Strategy for Infant and Young Child Feeding. Geneva: WHO, 2007. 